\title{
Nanopatterning of carbonaceous structures by field-induced carbon dioxide splitting with a force microscope
}

\author{
R. Garcia, ${ }^{1, a)}$ N. S. Losilla, ${ }^{1}$ J. Martínez, ${ }^{1}$ R. V. Martinez, ${ }^{1}$ F. J. Palomares, ${ }^{2}$ Y. Huttel, ${ }^{2}$ \\ M. Calvaresi, ${ }^{3}$ and F. Zerbetto ${ }^{3}$ \\ ${ }_{1}^{1}$ Instituto de Microelectrónica de Madrid, CSIC, Isaac Newton 8, Tres Cantos, 28760 Madrid, Spain \\ ${ }^{2}$ Instituto de Ciencia de Materiales de Madrid, CSIC, Cantoblanco, 28049 Madrid, Spain \\ ${ }^{3}$ Dipartimento di Chimica “G. Ciamician,” Universita' di Bologna, V. F. Selmi 2, 40126 Bologna, Italy
}

(Received 4 December 2009; accepted 8 March 2010; published online 5 April 2010)

\begin{abstract}
We report a tip-based nanofabrication method to generate carbon nanopatterns. The process uses the field-induced transformation of carbon dioxide gas into a solid material. It requires the application of low-to-moderate voltages $\sim 10-40 \mathrm{~V}$. The method allow us to fabricated sub-25 nm dots and it can be up scaled to pattern square centimeter areas. Photoemission spectroscopy shows that the carbon is the dominating atomic species of the fabricated structures. The formation of carbon nanostructures and oxides by atomic force microscope nanolithography expands its potential by providing patterns on the same sample with different chemical composition. (C) 2010 American Institute of Physics. [doi:10.1063/1.3374885]
\end{abstract}

The control and manipulation of electric fields at the nanoscale has enabled the emergence of a flexible atomic force microscope (AFM) nanolithography called local oxidation. ${ }^{1-4}$ This method is based on the field-induced activation of molecules within a water meniscus and the subsequent oxidation of the sample surface. ${ }^{5-7}$ Local oxidation nanolithography has been applied to fabricate nanoscale electronic devices, ${ }^{8,9}$ microelectromechanical systems ${ }^{10}$ or templates for the direct growth of single-molecule magnets ${ }^{11}$ or metallic nanoparticles. ${ }^{12,13}$ Local oxidation is compatible with imprinting techniques, thus enables the patterning of large areas has been demonstrated. ${ }^{4,14-16}$

The method has been extended to other liquids such as ethanol, ${ }^{17}$ octane, ${ }^{18,19}$ or hexadecane. ${ }^{7,20}$ Furthermore, fieldassisted nanopatterning has been performed on polymer surfaces $^{21}$ or by inducing the transfer of material from the tip to the surface. ${ }^{22}$

The interest in fabricating structures by AFM nanolithography of a chemical composition different from oxides, in particular carbon compounds, lies in its chemical complementarity. Thus heterogeneous nanopatterns could be generated where the chemical activity of a given region depends on the process used to fabricate the structure (oxidation versus carbon formation). Furthermore, the fabrication of nanopatterns of carbon materials could be simplified if the source of material comes directly from a gas. We hypothesize that the field-induced activation of liquid molecules can be extended to transform stable gases such as carbon dioxide in ambient conditions.

We report on the transformation of carbon dioxide into a solid carbonaceous material that can be deposited with nanoscale accuracy over a silicon surface. The process takes place at room temperature and uses low-to-moderate voltages $\sim 10-40$ V.) The process exploits the high electric fields that can be generated with a nanoscale asperity $(\sim 1-30 \mathrm{~V} / \mathrm{nm})$. The approach can be performed with a single nanoscale asperity (AFM) and with a macroscopic sur-

\footnotetext{
a) Author to whom correspondence should be addressed. Electronic mail: ricardo.garcia@imm.cnm.csic.es.
}

face patterned (stamp) with billions of nanoscale asperities.

The physical process [Fig. 1(a)] exploits the proximity effect between a flat surface and a sharp protrusion. This
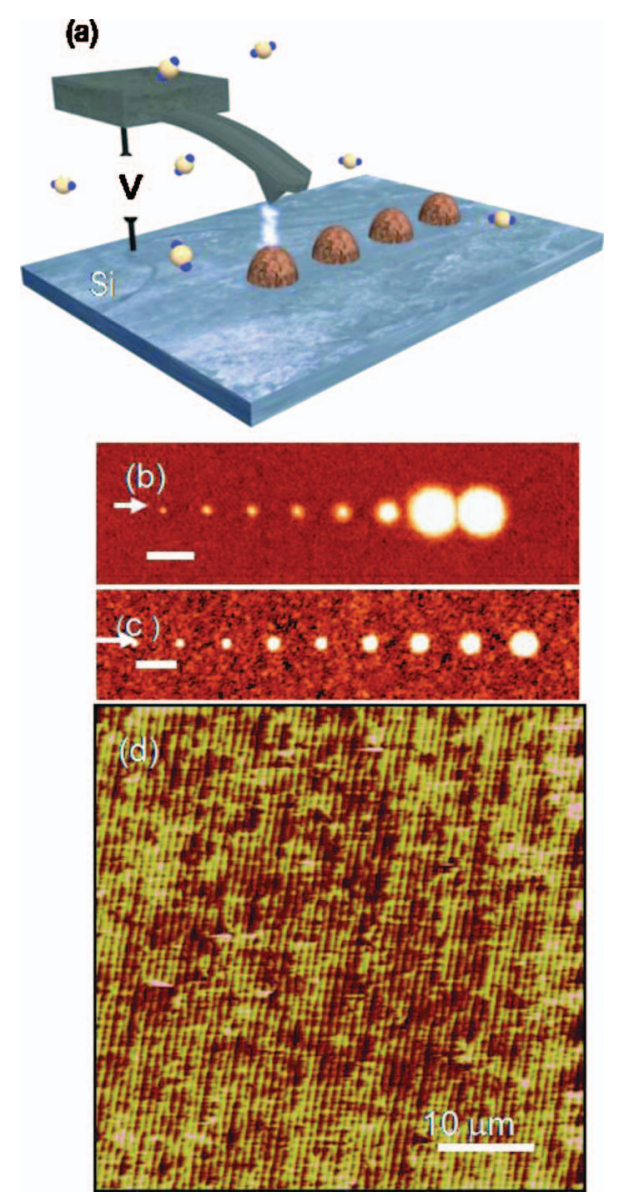

FIG. 1. (Color) (a) Schematics of the activation process with an AFM operated in amplitude modulation (noncontact regime). (b) AFM image of a sequence of dots generated by applying $21 \mathrm{~V}$ for, left to right, $0.1,0.5,1,10$, 50 , and $100 \mathrm{~ms}$, $1 \mathrm{~s}$ and $2 \mathrm{~s}$. (c) Sequence of dots generated by applying (from left to right) $20,22,24,26,28,30,34$, and $36 \mathrm{~V}$ pulses for $0.1 \mathrm{~ms}$. (d) AFM image of a carbonaceous film formed after the activation of $\mathrm{CO}_{2}$ gas with a stamp. Scale bar of $200 \mathrm{~nm}$ in (b) and (c). 


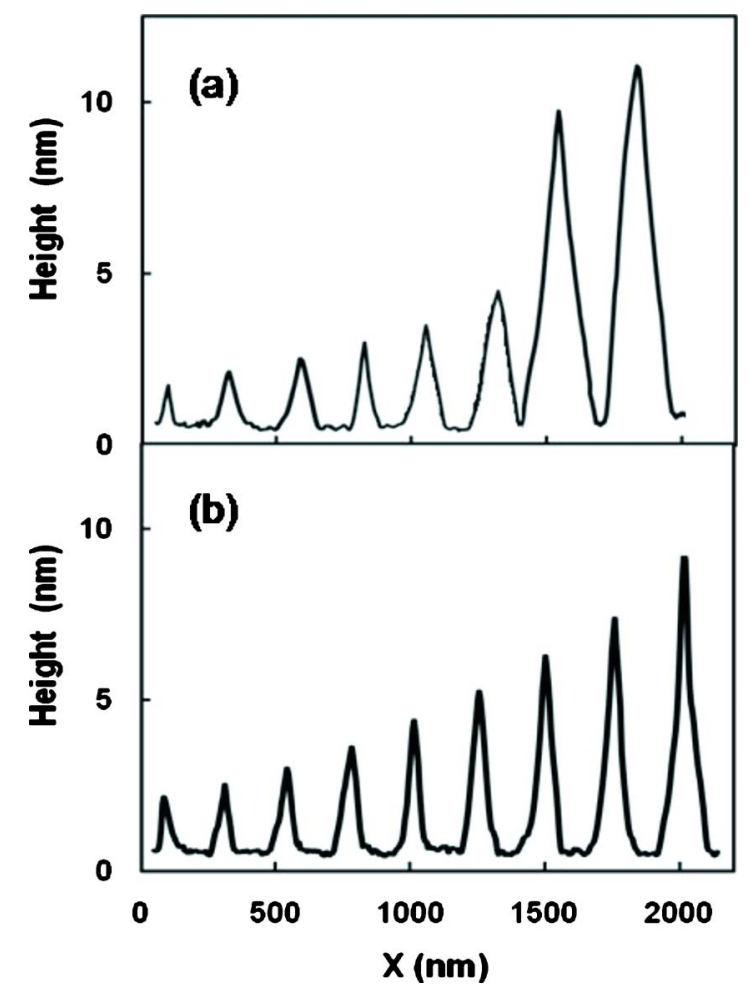

FIG. 2. (Color online) (a) Sequence of dots generated by applying $21 \mathrm{~V}$ for different pulse times [see caption of Fig. 1(b)]. (b) AFM image of a sequence of dots generated by the application of different voltages for $0.1 \mathrm{~ms}$ [see caption of Fig. 1(c)]. The arrows in Figs. 1(b) and 1(c) indicate the position of the cross-sections.

effect has two dominant electrostatic contributions arising from the length scale and the geometry of the interface. The electric field between a hyperboloidal tip and a flat conducting surface separated by a distance $L$ has two regimes. ${ }^{23-25}$ The field, $F_{t}$, at the apex of the protrusion is described as,

$$
F_{t}=\frac{1}{K_{t}} \frac{V}{R}
$$

where $R$ is the effective radius of the protrusion and $V$ is the applied voltage. At the flat surface, the field $F_{s}$ is,

$$
F_{s}=\frac{1}{K_{s}} \frac{V}{L}
$$

where $K_{t}$ and $K_{s}$ are geometrical factors. ${ }^{23,25}$ In the AFM experiments the field can be easily determined and varied by either modifying the distance or the voltage. The field has been changed from 0.1 to $30 \mathrm{~V} / \mathrm{nm}$.

Figure 1(a) shows the experimental set-up to activate carbon dioxide gas in a single asperity interface. The AFM tip is placed $2-5 \mathrm{~nm}$ above the silicon surface while the voltage pulse is applied. Figures 1(b) and 1(c) show a series of dots generated after the activation of $\mathrm{CO}_{2}$. Sub-25 nm dots are generated by applying short voltage pulses $(\sim 0.1 \mathrm{~ms})$.

Similarly to what happens with local oxidation experiments, ${ }^{15-17}$ the process can be upscaled by using a patterned stamp. Figure 1(d) shows a pattern formed after the activation of carbon dioxide with a gold-coated stamp patterned with an array of parallel lines $760 \mathrm{~nm}$ apart. The deposition occurs on the positively biased surface. The periodic pattern reflects the periodicity of the stamp and was gener-
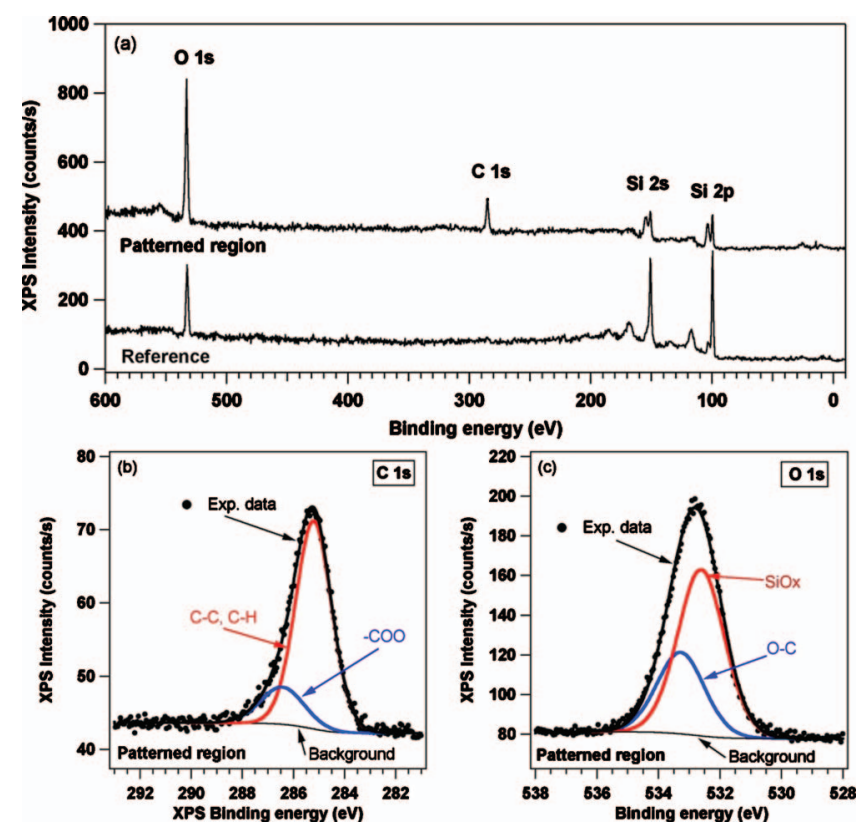

FIG. 3. (Color) (a) Wide X-ray photoemission spectrum performed on the carbonaceous film and the reference regions. (b) XPS C 1s core-level spectrum of a patterned region and the deconvolutions for the carbon-oxygen bonds (blue) and the $\mathrm{C}-\mathrm{C}$ and/or $\mathrm{C}-\mathrm{H}$ bonds (red). (c) XPS O 1s core-level spectrum of a patterned region and the deconvolutions for the carbonoxygen bonds (blue) and the $\mathrm{O}-\mathrm{Si}$ bonds (red). In (b) and (c) The filled dots joined by a continuous line are the experiments.

ated by applying a voltage pulse of $36 \mathrm{~V}$ for $60 \mathrm{~s}$. The fabricated structures act as resists with respect to hydrogen fluoride (HF) vapor exposure.

To optimize the conversion of $\mathrm{CO}_{2}$, we have varied the voltage pulse strength and duration with an amplitude modulation AFM operated in noncontact mode. ${ }^{26,27}$ Figure 2(a) shows the cross-sections of some dots obtained after the application of a voltage pulse of $21 \mathrm{~V}$ (pulse times ranging from $10^{-4}$ to $\left.2 \mathrm{~s}\right)$. For short pulse times $(\leq 0.1 \mathrm{~s})$, the dot height increases linearly with time. A sudden increase in size occurs at longer pulses. The diameter of the dots changes from 90 to $180 \mathrm{~nm}$ upon going from 0.1 to $2 \mathrm{~s}$. That implies a factor $\sim 10$ increase in the volume. The size dependence of the dots on voltage shows a monotonic increase [Fig. 2(b)]. The above experiment was performed at fixed pulse duration of $10^{-4} \mathrm{~s}$, while the voltage was changed from 20 to $36 \mathrm{~V}$ in $2 \mathrm{~V}$ steps.

The chemical composition has been characterized by photoemission spectroscopy experiments (XPS) at two different points. The first is located in a region where the activation of $\mathrm{CO}_{2}$ took place (patterned region). The second is in a region where no conversion was produced (reference region). The wide XPS spectrum of the patterned region reveals four different peaks [Fig. 3(a)]. The highest signals are centered at the $\mathrm{O} 1 \mathrm{~s}$ and $\mathrm{C} 1 \mathrm{~s}$ core-level binding energies. The other peaks correspond to the emission from the $\mathrm{Si} 2 \mathrm{~s}$ and $2 p$ core levels. The atomic concentrations extracted from the integrated intensities of $\mathrm{O} 1 \mathrm{~s}$ and $\mathrm{C} 1 \mathrm{~s}$ peaks in the patterned spectrum are, respectively, 2.2 and 6.4 times higher than in the reference. The presence of $\mathrm{C}$ and $\mathrm{O}$ on the top of the reference region is due to the air exposure of the sample surface. The spectroscopy data also indicate that the carbonaceous deposits contain six times more $\mathrm{C}$ than $\mathrm{O}$. At the same time, the atomic concentration of $\mathrm{Si}$ is smaller by a 
factor of 1.4 because the carbonaceous material on the top of the silicon surface reduces the Si signal.

The deconvolution of the higher energy resolution spectrum of the C 1s core level [Fig. 3(b)] reveals two dominant components at 285.2 and $286.7 \mathrm{eV}$. The binding energy of $285.2 \mathrm{eV}$ corresponds to either $\mathrm{C}-\mathrm{C}$ or to $\mathrm{C}-\mathrm{H}$ bonds in the $\mathrm{sp}^{3}$ tetrahedral configuration ${ }^{28,29}$ whereas the component located at $286.7 \mathrm{eV}$ corresponds to carbon-oxygen bonds. ${ }^{29}$ The amount of carbon in the patterns has increased $650 \%$ relative to the unpatterned regions. The increase in the carbon concentration is mostly due to $\mathrm{C}-\mathrm{C}$ bonds.

The resolution spectrum of the $\mathrm{O} 1 \mathrm{~s}$ core level has two components [Fig. 3(c)]. The first is centered at a binding energy of $532.6 \mathrm{eV}$. It shows the presence of $\mathrm{Si}$ oxides (mainly $\mathrm{SiO}_{2}$ ). The second component is centered at 533.2 $\mathrm{eV}$ and reveals the presence of $\mathrm{O}-\mathrm{C}$ bonds ${ }^{28}$ in agreement with the spectrum measured at the $\mathrm{C} 1 \mathrm{~s}$ level. The reference spectrum did not reveal the presence of $\mathrm{O}-\mathrm{C}$ bonds.

Quantum chemical calculations show that the purely electronic energy barrier for dissociation, $\Delta \mathrm{E}$, of one of the two OC $\cdots \mathrm{O}$ bonds is $\sim 6.1 \mathrm{eV}$, at zero field, and $\sim 3.25 \mathrm{eV}$ at $40 \mathrm{~V} / \mathrm{nm}$. At $20 \mathrm{~V} / \mathrm{nm}$ the barrier is $\sim 5.3 \mathrm{eV}$ and the reaction becomes exothermic with the products $(\mathrm{CO}, \mathrm{O})$ lower in energy than the reactants $\left(\mathrm{CO}_{2}\right)$. It can be proposed that the energy is spent to overcome the barrier for dissociation. As a function of the experimental conditions, or in other words of the field experienced by $\mathrm{CO}_{2}$ when it breaks apart, different amounts of energy are used.

The transformation of $\mathrm{CO}_{2}$ can be separated into three major steps. The first step is the capture of $\mathrm{CO}_{2}$ in the gap region between the two electrodes. The second step is the activation of the molecules, and third step is the formation of the carbonaceous deposit. The first step is a field-induced diffusion process. The electric field polarizes the molecule and the resulting dipole moment interacts with the field. The potential energy of a $\mathrm{CO}_{2}$ molecule under the influence of the field can be approximated as, ${ }^{30}$

$$
U=U_{0}-\frac{1}{2} \alpha F^{2},
$$

where $\alpha=2.93 \times 10^{-40} \mathrm{C}^{2} \mathrm{~m}^{2} \mathrm{~J}^{-1}$ is the static polarizability of the $\mathrm{CO}_{2}$ and $U_{0}$ is its energy in the absence of the field. As a consequence in a nonuniform field the molecules experience a polarization force toward the conductive surfaces. The high electric fields trap the $\mathrm{CO}_{2}$ between the conductive surfaces, and increase the gas density. We can estimate the change in pressure, $p$, between the electrodes as, $p=p_{0} \exp \left(\Delta U / k_{B} T\right)$, where $p_{0}$ is the pressure at zero field. For a field of $10 \mathrm{~V} / \mathrm{nm}$, we obtain $\Delta U=92 \mathrm{meV}$, which gives $p \approx 36 p_{0}(\mathrm{~T}=298 \mathrm{~K})$. Higher pressures imply higher collision rates between the gas molecules.

In a third step, the molecules diffuse to the solid where they become adsorbed. There the dissociated and activated molecules react with other molecules to form the carbon- aceous material. The resulting material is a carbon compound with the presence of some oxygen. This leads to the formation of carbonaceous nanopatterns that are resistant to HF etching.

We are very grateful to José M. Soler for their insightful and motivating comments. We acknowledge financial supports from the Ministerio de Ciencia, Investigación e Innovación (Grant Nos. MAT2009-08650; CTQ2007-31076-E; and MAT2008-06765).

${ }^{1}$ A. A. Tseng, A. Notargiacomo, and T. P. Chen, J. Vac. Sci. Technol. B 23, 877 (2005).

${ }^{2}$ X. N. Xie, H. J. Chung, C. H. Sow, and A. T. S. Wee, Mater. Sci. Eng. R. 54, 1 (2006).

${ }^{3}$ R. Garcia, R. V. Martinez, and J. Martinez, Chem. Soc. Rev. 35, 29 (2006).

${ }^{4}$ F. C. Simeone, C. Albonetti, and M. Cavallini, J. Phys. Chem. C 113, 18987 (2009).

${ }^{5}$ J. A. Dagata, F. Perez-Murano, C. Martín, H. Kuramochi, and H. Yokoyama, J. Appl. Phys. 96, 2386 (2004).

${ }^{6}$ M. Tello and R. Garcia, Appl. Phys. Lett. 79, 424 (2001).

${ }^{7}$ C. R. Kinser, M. J. Schmitz, and M. C. Hersam, Adv. Mater. 18, 1377 (2006)

${ }^{8}$ A. Fuhrer, S. Lüscher, T. Ihn, T. Heinzel, K. Esslin, W. Wegscheider, and M. Bichler, Nature (London) 413, 822 (2001).

${ }^{9}$ J. Martinez, R. V. Martinez, and R. Garcia, Nano Lett. 8, 3636 (2008).

${ }^{10}$ L. Pellegrino, M. Biasotti, E. Bellingeri, C. Bernini, A. S. Siri, and D. Marré, Adv. Mater. 21, 2377 (2009).

${ }^{11}$ R. V. Martínez, F. Garcia, R. Garcia, E. Coronado, A. Forment-Aliaga, F. M. Romero, and S. Tatay, Adv. Mater. 19, 291 (2007).

${ }^{12}$ R. Maoz, E. Frydman, S. R. Cohen, and J. Sagiv, Adv. Mater. 12, 725 (2000).

${ }^{13}$ S. Liu, R. Maoz, and J. Sagiv, Nano Lett. 4, 845 (2004).

${ }^{14}$ S. Hoeppener, R. Maoz, and J. Sagiv, Nano Lett. 3, 761 (2003).

${ }^{15}$ A. Zeira, D. Chowdhury, R. Maoz, and J. Sagiv, ACS Nano 2, 2554 (2008).

${ }^{16}$ N. S. Losilla, J. Martinez, and R. Garcia, Nanotechnology 20, 475304 (2009).

${ }^{17}$ M. Tello and R. Garcia, Appl. Phys. Lett. 83, 2339 (2003).

${ }^{18}$ I. Suez, S. A. Backer, and J. M. J. Frechet, Nano Lett. 5, 321 (2005).

${ }^{19}$ R. V. Martínez, N. S. Losilla, J. Martinez, Y. Huttel, and R. Garcia, Nano Lett. 7, 1846 (2007).

${ }^{20}$ I. Suez, M. Rolandi, S. A. Backer, A. Scholl, A. Dogan, D. Okawa, A. Zettl, and J. M. J. Frechet, Adv. Mater. 19, 3570 (2007).

${ }^{21}$ M. Rackaitis, D. Kashyn, T. Hirano, and S. F. Lyuksyutov, Appl. Phys. Lett. 93, 183110 (2008).

${ }^{22}$ J.-F. Liu and G. P. Miller, Nanotechnology 20, 055303 (2009).

${ }^{23}$ R. Gomer, Field Emission and Field Ionization (American Institute of Physics, New York, 1993).

${ }^{24}$ H. J. Kreuzer, Surf. Sci. 246, 336 (1991).

${ }^{25}$ G. Mesa, E. Dobado-Fuentes, and J. J. Saenz, J. Appl. Phys. 79, 39 (1996).

${ }^{26}$ A. Raman, J. Melcher, and R. Tung, Nano Today 3, 20 (2008).

${ }^{27}$ M. Tello, R. Garcia, J. A. Martín-Gago, N. F. Martinez, M. S. MartinGonzalez, L. Aballe, A. Baranov, and L. Gregoratti, Adv. Mater. 17, 1480 (2005)

${ }^{28}$ R. Haerle, E. Riedo, A. Pasquarello, and A. Baldereschi, Phys. Rev. B 65, 045101 (2001).

${ }^{29}$ T. I. T. Okpalugo, P. Papakonstantinou, H. Murphy, J. McLaughlin, and N. Brown, Carbon 43, 153 (2005).

${ }^{30}$ G. Maroulis and A. J. Thakkar, J. Chem. Phys. 93, 4164 (1990). 